\title{
NavOptim: \\ On the Possibility of Minimising Navigation Effort
}

\author{
Xiaoying Kong and David Lowe \\ University of Technology, Sydney, P.O. Box 123, Broadway, NSW, Australia \\ \{xiaoying.kong,david.lowe\}@uts.edu.au
}

\begin{abstract}
Web applications have rapidly become critical to the interaction that organisations have with their external stakeholders. A major factor in the effectiveness of this interaction is the ease with which users can locate information and functionality which they are seeking. Effective design is complicated by the multiple purposes and users which Web applications support. In our earlier work we described a model for evaluating the weighted effort required of users. In this paper we describe an approach to minimizing this navigational effort.
\end{abstract}

\section{Introduction}

Over the last decade the Web has become a key vehicle for accessing organisational applications. These applications often provide crucial business or government services, and hence the quality of the user interaction and the extent to which users are able to achieve their goals is vital to the success of the systems. Given this, effective design of the web application interface is crucial. This in turn raises the issue of what is actually meant by "effective design"? Effectiveness can be defined in terms of the ability to support the users' goals - yet for Web applications these goals are typically both complex and diverse, with different users having different expectations and objectives. Optimising the design becomes a difficult activity involving trade-offs of multiple constraints. Despite this the design of navigation structures is rarely treated as an optimization problem.

\section{Related Work}

Numerous approaches have been developed for performing the design of the navigational structure of Web systems. Early approaches in this area tended to emerge from the Hypertext community and evolved out of work on Entity-Relationship modelling (e.g. RMM [1]) or object-oriented modelling (e.g. OOHDM [2]). Other approaches have included EORM [3] and WSDM [4], and more recently WebML [5]. The focus of these approaches is very much on modelling the underlying content, the user viewpoints onto this content, and the navigational structures that interlink the content. They have however usually undertaken the navigation design based on a subjective view of how users are likely to want to interact with the information. In most cases this is not well informed by the underlying requirements that drive this architecture. They do not typically provide any formal way to ensure that the navigation structures are theoretically optimal. There has also been substantial research investigating navi- 
gation design based on either likely usage patterns or an analysis of actual usage e.g. user-centred design [6]. Again, however, this does not guarantee a theoretical optimisation.

\section{Minimising Navigational Effort}

In each of the above cases the approach is focussing on subjective analysis and refinement of the navigational structure. We argue that this is inappropriate, since the design subjectivity lies not in the navigational structure, but rather in the significance of the various information and services that are provided to users and the design choices about the usage patterns which we wish the system to support. Once this is known we ought to be able to design the navigational structure which provides the theoretical minimum navigational effort required by users in accessing these services and information - assuming we have a reliable measure of navigational effort.

In our earlier work we described a model of the relationship between the intended user tasks, the navigational structure and the resultant overall navigational effort. The overall weighted navigational effort for a site can be modelled as:

$$
H_{\text {sys }}=-\sum_{j=1}^{n}\left[\operatorname{Sgnf}(j) *\left(\sum_{i=1}^{m} p_{i} \log p_{i}\right)\right] \quad p_{i}=\frac{\operatorname{Smt} \operatorname{Coh}\left(V_{i}, V_{\text {Taskcase }}\right)}{\sum_{j=1}^{m} \operatorname{SmtCoh}\left(V_{j}, V_{\text {Taskcase } 1}\right)}, i=1: m
$$

where $\operatorname{Sgnf}(j)$ is the significance of a particular task $j$, and $\operatorname{SmtCoh}$ provides a measure of the semantic cohesion between two information vectors, and $V$ is an information vector. Details on this model are given in [7]. Whilst this equation provides a basis for evaluating and comparing designs, it still does not allow us to determine the theoretical optimum structure. We can compare this to data compression algorithms in information theory: if we have a message " $M s g$ " which consists of $M$ different symbols, each with a given probability of appearing in the message $M s g$, then we can then identify the theoretical minimum for the average number of bits to code each character in the string. Further, techniques such as Huffman coding [8] provide an algorithm that determines the the coding for each symbol in order to achieve this minimum.

We would like to be able to identify an equivalent algorithm for determining the navigational structure which achieves a theoretically minimum navigational effort. We have however found this problem to be intractable. This is for several reasons. The first is that whilst the Huffman coding tree is a binary tree, the NavOptim navigation tree is an $n$-ary tree where $n$ varies in each branch of the tree. Even more problematic is that whilst the characters in the Huffman coding are independent, the same is not true of the nodes in the NavOptim tree. The individual entropies of each navigational path are related to the location of all nodes within the tree. In other words, whilst there will indeed be a thoereticaly optimal navigation structure for a given set of task cases, the algorithmic determination of this structure is an intractable problem. This then leads to the question of whether we can identify a (sub-optimal) approximation to the theoretically optimal navigation tree?

Given the intractability of finding a navigational design algorithm which can be mathematical proven to result in the minimization of equation 1 , we can turn our 
attention to approaches which approximate an optimal design, but which are strongly guided by the underlying theory. We begin by analyzing the mathematical models captured by equation 1 . The first observation is that the effort is strongly coupled to the overall navigational depth of specific content. As content is located deeper within the navigational tree, the navigational effort to locate it will typically increase (all other factors being equal). The second is that the effort is strongly related to the semantic cohesion. As we create stronger cohesion within branches of the tree, we enable clearer navigation choices and hence reduced entropy and effort. Figure 1 illustrates this issue graphically for different content clusterings, where the spatial positioning of the pages represents the semantic distance between the pages, and the highlighted page is the target of a given task.

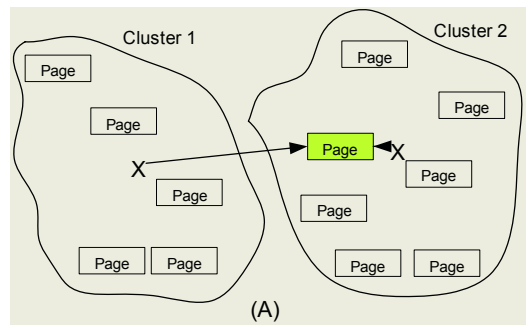

(A)

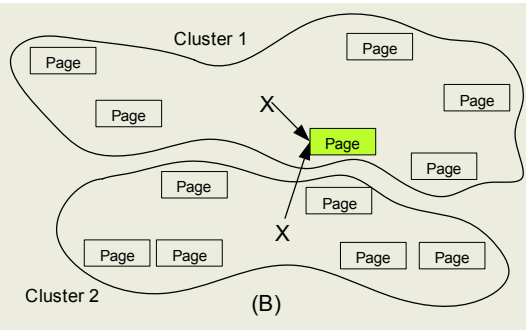

(B)

Fig. 1. Example clustering

\section{Conclusions}

In this paper we have discussed issues in the design of navigational structures based on the optimisation of a navigational effort metric weighted by the task significances. Subsequent work, to be published elsewhere, describes an approach which will lead to a reduction in the average effort required to locate information or services within websites. We are also investigating tools to simulate different websites to see how they are optimised using our approach, followed by subjective evaluation to determine whether this does indeed lead to qualitative improvements.

\section{References}

1. T. Isakowitz, E. Stohr, and P. Balasubramanian, "RMM: A Methodology for Structured Hypermedia Design," Communications of the ACM, vol. 38, pp. 34-44, 1995.

2. D. Schwabe and G. Rossi, "Developing Hypermedia Applications using OOHDM," presented at Workshop on Hypermedia Development Processes, Methods and Models (Hypertext'98), Pittsburgh, USA, 1998.

3. D. Lange, "An Object-Oriented Design Method for Hypermedia Information Systems," presented at HICSS-27: Proc of the Twenty Seventh Hawaii International Conference on System Sciences, Maui, Hawaii, 1994.

4. O. De Troyer and C. Leune, "WSDM: A user-centered design method for Web sites," presented at 7th International World Wide Web Conference, Brisbane, Aust, 1997.

5. S. Ceri, P. Fraternali, and A. Bongio, "Web Modeling Language (WebML): a modeling language for designing Web sites," presented at Proceedings of WWW9 Conference, Amsterdam, 2000. 
6. D. A. Norman and S. W. Draper, User-Centered Design. Hillsdale, N.J.: Lawrence Erlbaum Assoc, 1986.

7. D. Lowe and X. Kong, "NavOptim Coding: Supporting Website Navigation Optimisation using Effort Minimisation," presented at 2004 IEEE/WIC/ACM International Conference on Web Intelligence, Beijing, China, 2004.

8. D. A. Huffman, "A method for the construction of minimum redundancy codes," Proc. IRE, vol. 40, pp. 1098-1101., 1952. 